\title{
BMJ Open Are there researcher allegiance effects in diagnostic validation studies of the PHQ-9? A systematic review and meta- analysis
}

\author{
Laura Manea, ${ }^{1,2}$ Jan Rasmus Boehnke, ${ }^{3}$ Simon Gilbody, ${ }^{1,2}$ Andrew S Moriarty, ${ }^{2}$ \\ Dean McMillan ${ }^{1,2}$
}

To cite: Manea L, Boehnke JR Gilbody S, et al. Are there researcher allegiance effects in diagnostic validation studies of the PHQ-9? A systematic review and meta-analysis. BMJ Open 2017;7:e015247. doi:10.1136/ bmjopen-2016-015247

- Prepublication history and additional material for this paper are available online. To view, please visit the journal (http:// dx.doi.org/10.1136/bmjopen2016-015247).

Received 23 November 2016 Revised 21 July 2017 Accepted 25 July 2017

\section{CrossMark}

${ }^{1}$ Deparment of Health Sciences, University of York, York, UK ${ }^{2}$ Hull York Medical School, University of York, York, United Kingdom

${ }^{3}$ Dundee Centre for Health And Related Research, University of Dundee, Dundee, United Kingdom

Correspondence to

Dr Laura Manea;

laura.manea@york.ac.uk

\section{ABSTRACT}

Objectives To investigate whether an authorship effect is found that leads to better performance in studies conducted by the original developers of the Patient Health Questionnaire (PHQ-9) (allegiant studies).

Design Systematic review with random effects bivariate diagnostic meta-analysis. Search strategies included electronic databases, examination of reference lists and forward citation searches.

Inclusion criteria Included studies provided sufficient data to calculate the diagnostic accuracy of the PHQ-9 against a gold standard diagnosis of major depression using the algorithm or the summed item scoring method at cut-off point 10.

Data extraction Descriptive information, methodological quality criteria and $2 \times 2$ contingency tables.

Results Seven allegiant and 20 independent studies reported the diagnostic performance of the PHQ-9 using the algorithm scoring method. Pooled diagnostic $\mathrm{OR}$ (DOR) for the allegiant group was 64.40, and 15.05 for non-allegiant studies group. The allegiance status was a significant predictor of DOR variation $(p<0.0001)$. Five allegiant studies and 26 non-allegiant studies reported the performance of the PHQ-9 at recommended cut-off point of 10. Pooled DOR for the allegiant group was 49.31, and 24.96 for the non-allegiant studies. The allegiance status was a significant predictor of DOR variation $(p=0.015)$. Some potential alternative explanations for the observed authorship effect including differences in study characteristics and quality were found, although it is not clear how some of them account for the observed differences.

Conclusions Allegiant studies reported better performance of the PHQ-9. Allegiance status was predictive of variation in the DOR. Based on the observed differences between independent and non-independent studies, we were unable to conclude or exclude that allegiance effects are present in studies examining the diagnostic performance of the PHQ-9. This study highlights the need for future meta-analyses of diagnostic validation studies of psychological measures to evaluate the impact of researcher allegiance in the primary studies.

Research on allegiance effects has a long tradition in psychotherapy research. In this
Strengths and limitations of this study

- An original study-the first meta-analysis of diagnostic validation studies of psychological measures to evaluate the impact of researcher allegiance.

- Using rigorous methodology-strict inclusion/ exclusion and quality assessment criteria.

- We found that the allegiance effect was a significant predictor of the variation of the diagnostic OR in the meta-regression analysis.

- Substantial variability observed in methodological quality of included studies.

- Based on the observed methodological differences between the independent and non-independent studies, we were unable to conclude or exclude that allegiance effects are present in studies examining the diagnostic performance of the Patient Health Questionnaire (PHQ-9).

context, allegiance describes the phenomenon that researchers and clinicians who developed a treatment approach or are for other reasons invested in it tend to find larger effect sizes in favour of their treatment than for comparison groups. ${ }^{1}$ This finding has been extensively replicated ${ }^{2}{ }^{3}$ and is also robust when the quality of research is controlled for. Researcher allegiance is subject of ongoing debates about the design of efficacy studies as well as implications for policy. ${ }^{245}$ Researcher allegiance is also discussed widely in the literature on experimental as well as evaluation research. ${ }^{6}$ Since the motivational underpinnings of allegiance effects are potentially far more ingrained into human behaviour and decision making than previously thought, ${ }^{7}$ they may occur commonly in clinical research in general.

Although it has been suggested that allegiance effects may play a role in the validation of psychological screening and case-finding tools (eg, O'Shea et al., in press), systematic 
evaluations of this hypothesis are rare and studies that acknowledge potential allegiance effects in such studies mainly come from forensic psychology and psychiatry backgrounds. ${ }^{8-11}$ Diagnostic validation studies are geared at establishing the sensitivity and specificity of a screening or case-finding tool, which is used in practice to differentiate cases from non-cases or to decide about whether further assessment or treatment is indicated or will be offered. An allegiance effect in such studies would be seen in systematically higher sensitivities or specificities if the original author(s) is (are) part of the team of such a study. Such a bias would have a deleterious affect on practice through promising overoptimistic accuracy of the screening or case-finding tool or in evaluating the cost-effectiveness of the measure in a screening or casefinding context.

The depression module of the Patient Health Questionnaire (PHQ-9) is a widely used depression-screening instrument in non-psychiatric settings. The PHQ-9 was developed by a team of researchers, with its development underwritten by an educational grant from Pfizer US Pharmaceuticals. ${ }^{12}$ The PHQ-9 can be scored using different methods, including an algorithm based on Diagnostic and Statistical Manual of Mental Disorders (DSM)-IV criteria and a cut-off based on summed-item scores. The psychometric properties of these two approaches have been summarised in two recently published meta-analyses. ${ }^{13} 14$ The goal of the current review is to investigate, based on an established database of PHQ-9 diagnostic validation studies, ${ }^{13}{ }^{14}$ whether an allegiance effect is found that leads to an increased sensitivity and specificity in studies that were conducted by researchers closely connected to the original developers of the instrument.

\section{METHODS}

\section{Study selection}

Similar search strategies were used in both systematic reviews (for full details, please see Manea et al and Moriarty $\left.e t \quad a l^{13}{ }^{14}\right)$. Embase, Medline and PsycINFO were searched from 1999 (when the PHQ-9 was first developed) to August 2013 and September 2013, respectively, using the terms 'PHQ-9', 'PHQ', 'PHQ\$' and 'patient health questionnaire'. The search strategy is presented in online supplementary appendix 1 . The reference lists of studies fitting the inclusion criteria were manually searched and a reverse citation search in Web of Science was performed. The authors of unpublished studies were contacted and conference abstracts were reviewed in an attempt to minimise publication bias.

The following inclusion-exclusion criteria were used:

Population: adult population. Instrument: studies that used the PHQ-9. Comparison (reference standard): the accuracy of the PHQ-9 had to be assessed against a recognised gold-standard instrument for the diagnosis of either DSM or International Classification of Disease (ICD) criteria for major depression. Studies were included if the diagnoses were made using a standardised diagnostic structured interview schedule (eg, Mini International Neuropsychiatric Interview (MINI), Structured Clinical Interview for DSM Disorders (SCID)). Unguided clinician diagnoses with no reference to a standard structured diagnostic schedule or comparisons of the PHQ-9 with other self-report measures were excluded. Studies were also excluded if the target diagnosis was not major depressive disorder (MDD, eg, any depressive disorder). Outcome: studies had to report sufficient information to calculate a $2 \times 2$ contingency table for the algorithm or the recommended cut-off point 10. Study design: any design. Additional criterion: we avoided double counting of evidence by ensuring that only one study of those that reported overlapping datasets in different journals were included in the meta-analysis. Citations with overlapping samples were examined to establish whether they contained information relevant to the research question that was not contained in the included report.

\section{Quality assessment}

Quality assessment was performed using the Quality Assessment of Diagnostic Accuracy Studies (Revised) (QUADAS-2) tool, a tool for evaluating the risk of bias and applicability of primary diagnostic accuracy studies when conducting diagnostic systematic reviews. ${ }^{15}$ It covers the areas of patient selection, index test, reference standard and flow and timing. ${ }^{16}$ This tool was adapted for the two reviews and quality assessments were carried out by two independent reviewers for all studies included in the reviews.

\section{Data synthesis and statistical analysis}

We constructed $2 \times 2$ tables for cut-off point $10^{14}$ and the algorithm scoring method. ${ }^{13}$ Pooled estimates of sensitivity, specificity, positive/negative likelihood ratios and diagnostic ORs (DOR) were calculated using random effects bivariate meta-analysis. ${ }^{17}$ Heterogeneity was assessed using $\mathrm{I}^{2}$ for the DOR, an estimate of the proportion of study variability that is due to between-study variability rather than sampling error. We considered values of $\geq 50 \%$ to indicate substantial heterogeneity. ${ }^{18}$ Summary receiver operating characteristic curves (sROC) were constructed using the bivariate model to produce a $95 \%$ confidence ellipse within ROC space. ${ }^{19}$ Each data point in the sROC space represents a separate study, unlike a traditional ROC plot, which explores the effect varying thresholds on sensitivity and specificity in a single study.

We undertook a meta-regression analysis of logit DOR using research allegiance as covariate in the meta-regression model. ${ }^{20}{ }^{21}$ Analyses were conducted using STATA V.12, with the metan, metandi and metareg user-written commands.

\section{Allegiance rating}

We rated authorship on a paper if any of the developers of the PHQ-9-Kurt Kroenke, MD, Robert L Spitzer, MD and Janet BW Williams-as an indicator of potential allegiance. We also rated as evidence of allegiance as 
acknowledged collaborations with the developers of the PHQ-9, even if they were not listed as coauthors or if the authors acknowledged funding from Pfizer to conduct the study.

\section{RESULTS}

\section{Overview of included studies}

Thirty-one studies reported the diagnostic properties of the PHQ-9 at cut-off point 10 or above and were included in this analysis. ${ }^{14}$ Twenty-seven studies were included in the algorithm review. ${ }^{13}$ The study selection flow charts can be found in online supplementary appendix 2 (figures 1 and 2). The characteristics of these studies are reported in tables 1 and 2 and the results of the methodological assessment are presented in tables 3 and 4 .

\section{Algorithm scoring method \\ Descriptive characteristics}

The descriptive characteristics of the included studies are presented in table 1 . Seven individual studies that reported the diagnostic performance of the PHQ-9 using the algorithm scoring method were coauthored by the original developers of the PHQ-9, ${ }^{22-26}$ specifically acknowledged one of the developers and support by an educational grant from Pfizer USA, ${ }^{27}$ or were coauthored by the first author of a previous study that had also been coauthored by one of the developers. ${ }^{28}$ Twenty non-allegiant studies reported the diagnostic properties of the PHQ-9 using the algorithm scoring method.

Three $(43 \%, 3 / 7)$ of the allegiant studies were conducted exclusively in hospital settings. ${ }^{22} 2628$ The remaining four studies $(67 \%, 4 / 7)$ were conducted in different settings or non-exclusively hospital settings: one in primary care ${ }^{25}$ and three in mixed settings: psychosomatic walk in clinics and family practices ${ }^{23}$, , outpatient clinics and family practices ${ }^{24}$ and primary care and hospital settings. ${ }^{27}$ In the non-allegiant group, 13 (65\%, $13 / 20)$ studies were conducted in hospital settings. ${ }^{29-41}$ Of the remaining seven studies, six were conducted in primary care settings ${ }^{42-47}$ and one in a community sample. ${ }^{48}$

In both groups (non-allegiant and allegiant studies), the majority of studies validated a translated version of the PHQ-9. Two of the studies authored by developers $(28 \%, 2 / 7),{ }^{25}{ }^{26}$ and eight $(40 \%, 8 / 20)$ allegiant studies $^{29} 3037-404248$ were conducted in English.

The mean prevalence of MDD in the group of allegiant studies was $13.4 \%$ (range $6.1 \%-29.2 \%$ ); in the non-allegiant group it was $15.5 \%$ (range $3.9 \%-32.4 \%$ ). The mean age of patients in the PHQ-9 developers group was 45.7; all but one study had a mean age in the range of $40-50$ years. In the non-allegiant group, the mean age was 54.6 (range

\footnotetext{
${ }^{\mathrm{i}}$ This study provided separate estimates for the two settings in which it was conducted; therefore separate psychometric estimates were generated for each sample for both algorithm scoring method and summed items scoring method at cut-off point 10 (see below).
}

29.3-75.0), with almost half (8) of the studies reporting a mean age of over 60 . The percentage of females in the PHQ-9 developers was $56.8 \%$ (range $28.6 \%-67.8 \%$ ) and in the non-allegiant group was $59.1(18 \%-100 \%)$.

All allegiant studies used a self-reported PHQ-9, whereas in seven non-allegiant studies $(30 \%, 6 / 20)$ the PHQ-9 was administered by a researcher. ${ }^{30-33} 4348$ Apart from Muramatsu et al., all allegiant studies used the SCID as a gold standard ${ }^{27}$; the non-allegiant studies used a wider range of gold standards including SCAN, CIDI, MINI and C-DIS, although the SCID was also frequently used by the independent studies as well (45\%, 9/20 studies).

Four out of the seven allegiant studies $(57 \%)$ did not include a conflict of interest statement. ${ }^{22} 232527$ Also, four $(57 \%)$ of the allegiant studies acknowledged funding from Pfizer. ${ }^{23-25} 27$ Only one study ${ }^{27}$ acknowledged the collaboration with one of the developers of the PHQ-9.

Of the non-allegiant studies, $12(60 \%)$ did not include a conflict of interest statement. ${ }^{29-32}$ 35-37 39 44-46 48 It appears that newer studies were more likely to include a conflict of interest statement, which may reflect a recent change in reporting. Funding was acknowledged by most studies $(18 / 20)$ and most received funding from academic or/ and health research institutions. Two studies received funding from pharmaceutical companies-Lundbeck ${ }^{43}$ and Pfizer $^{35}$ and one study acknowledged that Pfizer Italia provided the Italian version of PHQ-9 and gave the authors permission to use it. ${ }^{36}$

\section{Diagnostic test accuracy}

Pooled sensitivity and specificity was calculated separately for the non-allegiant and allegiant studies. Pooled sensitivity for the allegiant studies of the PHQ-9 was 0.77 (95\% CI 0.70 to 0.84 ), pooled specificity was 0.94 (95\% CI 0.90 to 0.97 ) and the pooled DOR was 64.40 (95\% CI 34.15 to 121.43$)$. Heterogeneity was high $\left(I^{2}=78.9 \%\right)$. Figure 1 represents the sROCs for this set of studies.

Pooled sensitivity for the non-allegiant studies was lower compared with the developer authored studies group at 0.48 (95\% CI 0.41 to 0.91 ), pooled specificity was the same at 0.94 (95\% CI 0.91 to 0.95 ). The pooled DOR was approximately four times lower at 15.05 (95\% CI 11.03 to 20.52) (see figure 1). Heterogeneity was substantial at $\mathrm{I}^{2}=68.1 \%$.

The meta-regression analysis for algorithm studies with non-allegiant status as the predictor of the DOR showed that non-allegiant status was a significant predictor of the DOR $(\mathrm{p}<0.0001)$ and explained a substantial amount of the observed heterogeneity $(51.5 \%)$.

\section{Quality assessment}

The results of the quality assessment using QUADAS-2 are given in table 3 for the studies reporting on the diagnostic performance of the algorithm scoring method. In the patient selection domain, more non-allegiant studies $(65 \%, 13 / 20)$ than allegiant $(29 \%, 2 / 7)$ met the criterion for consecutive referrals. There were no marked differences on the other two criteria in this domain (avoid 


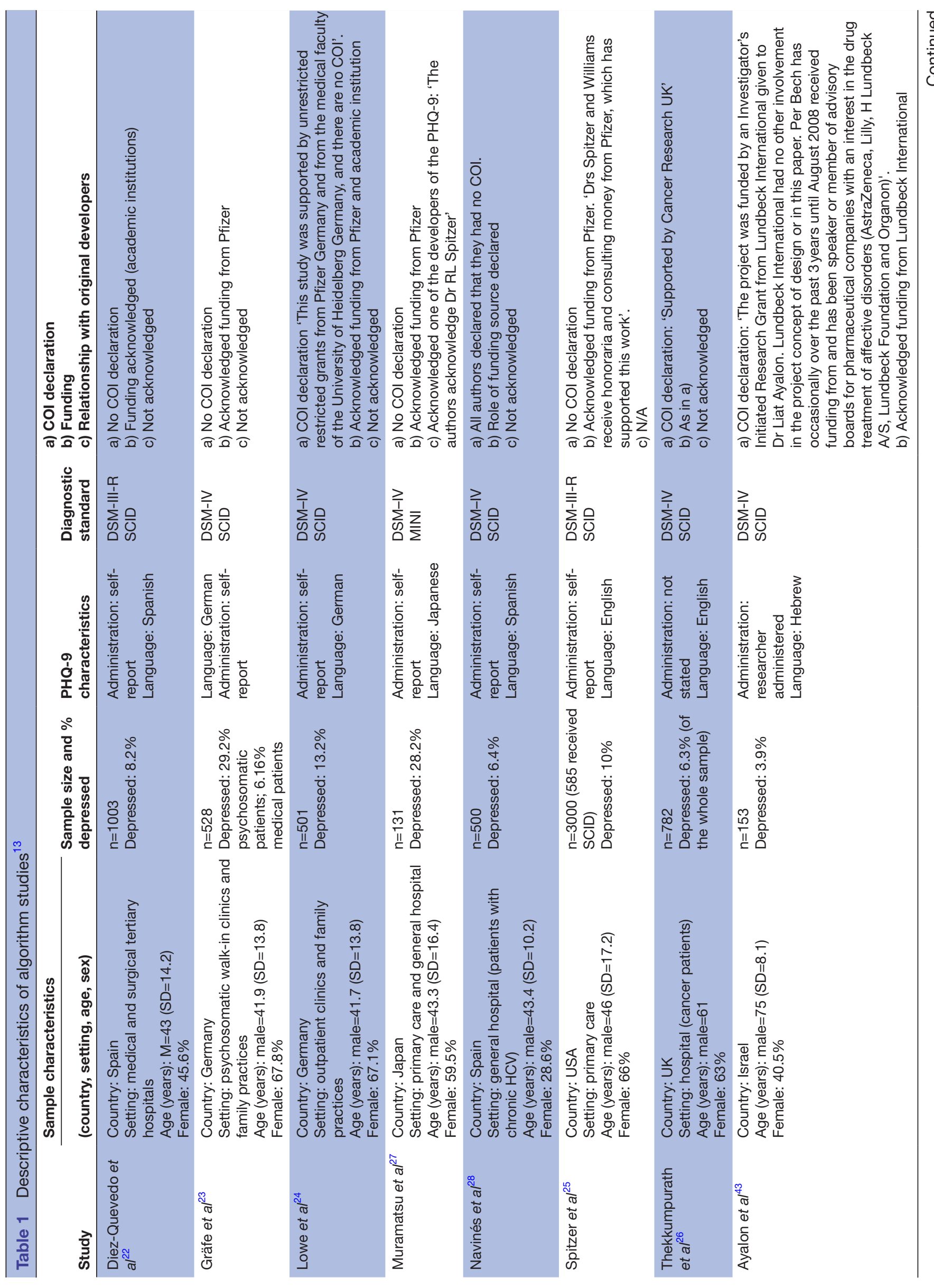




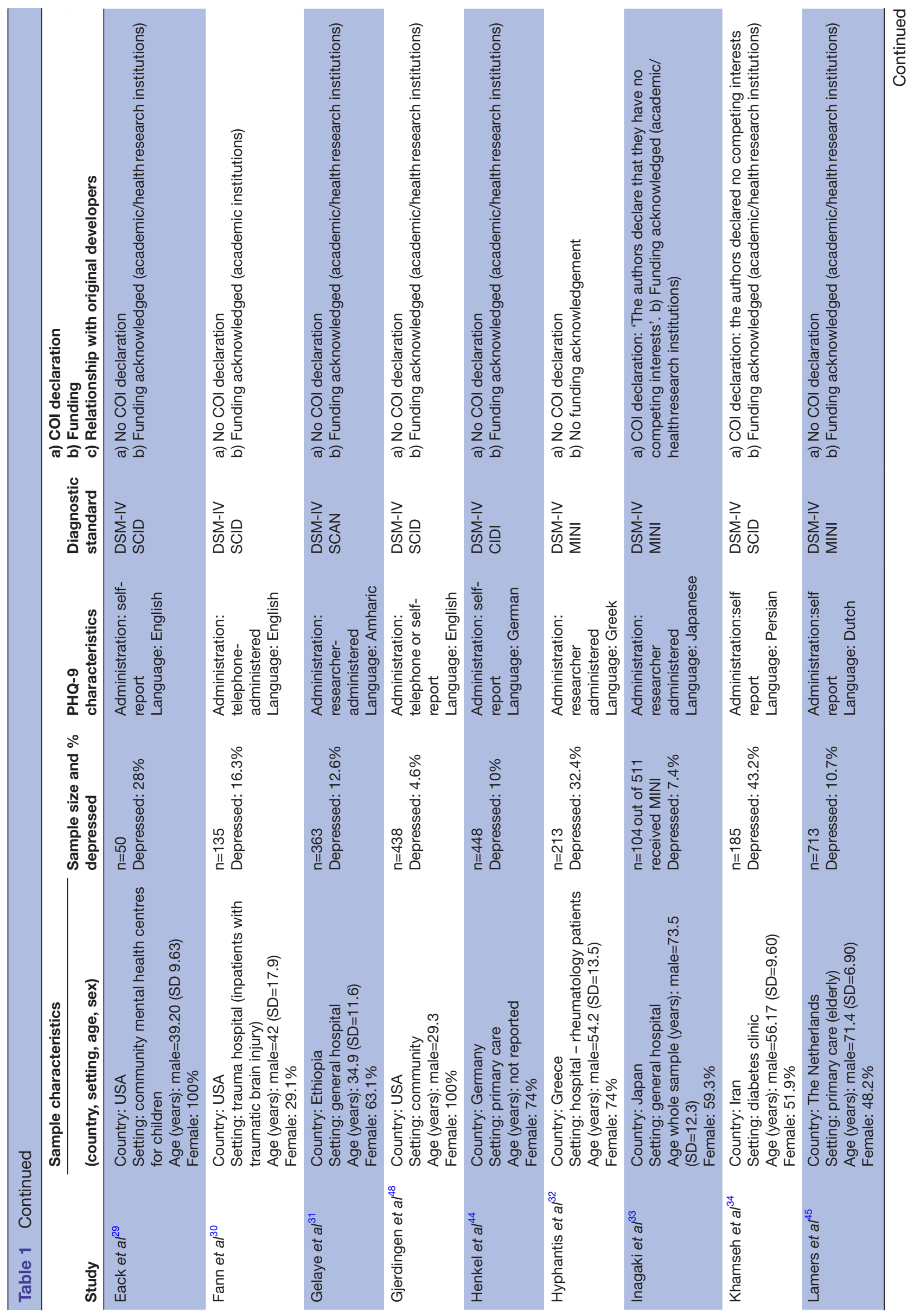




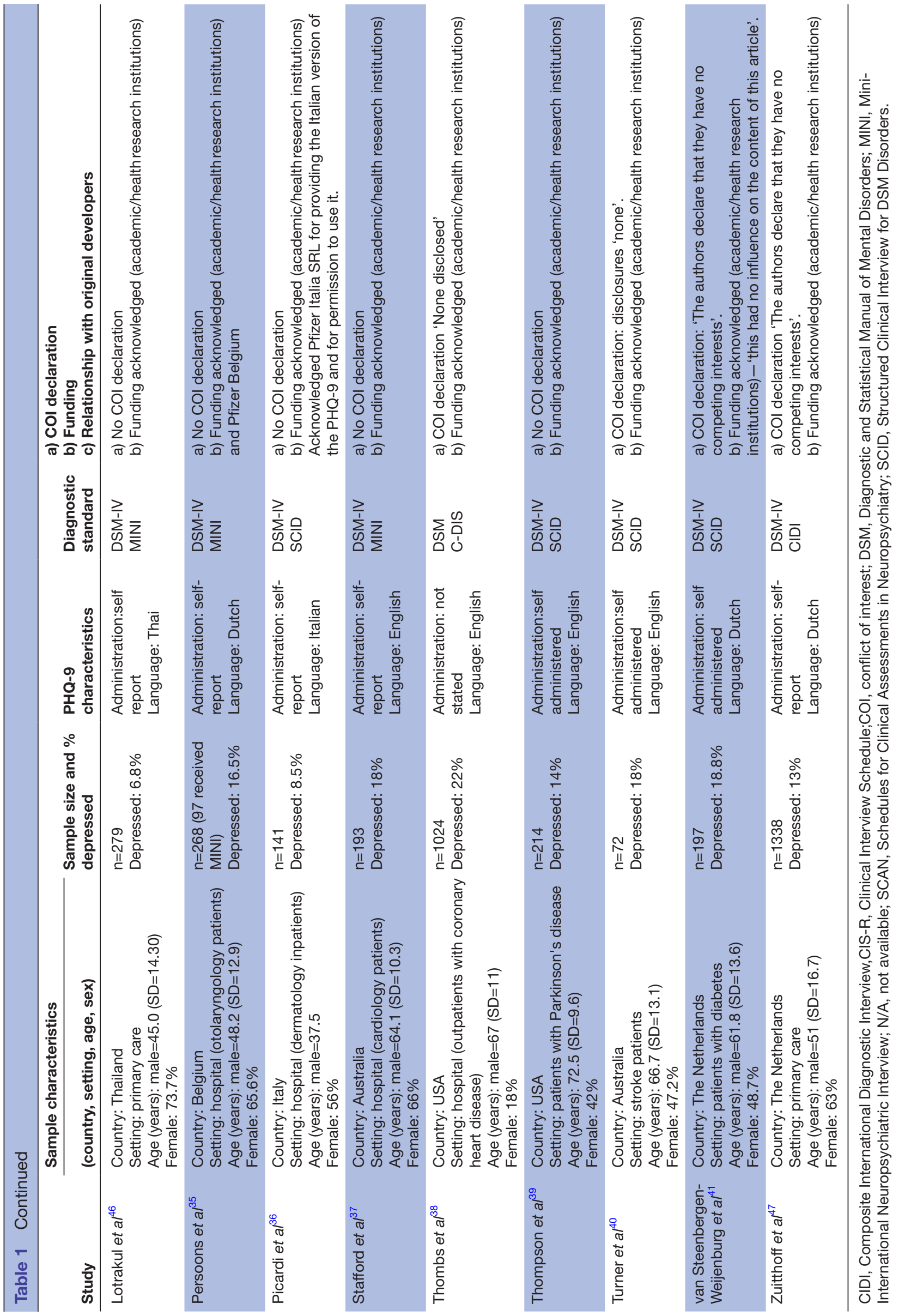

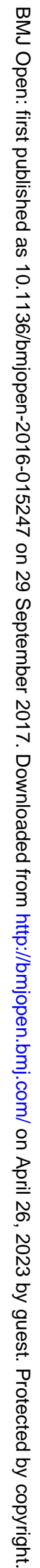




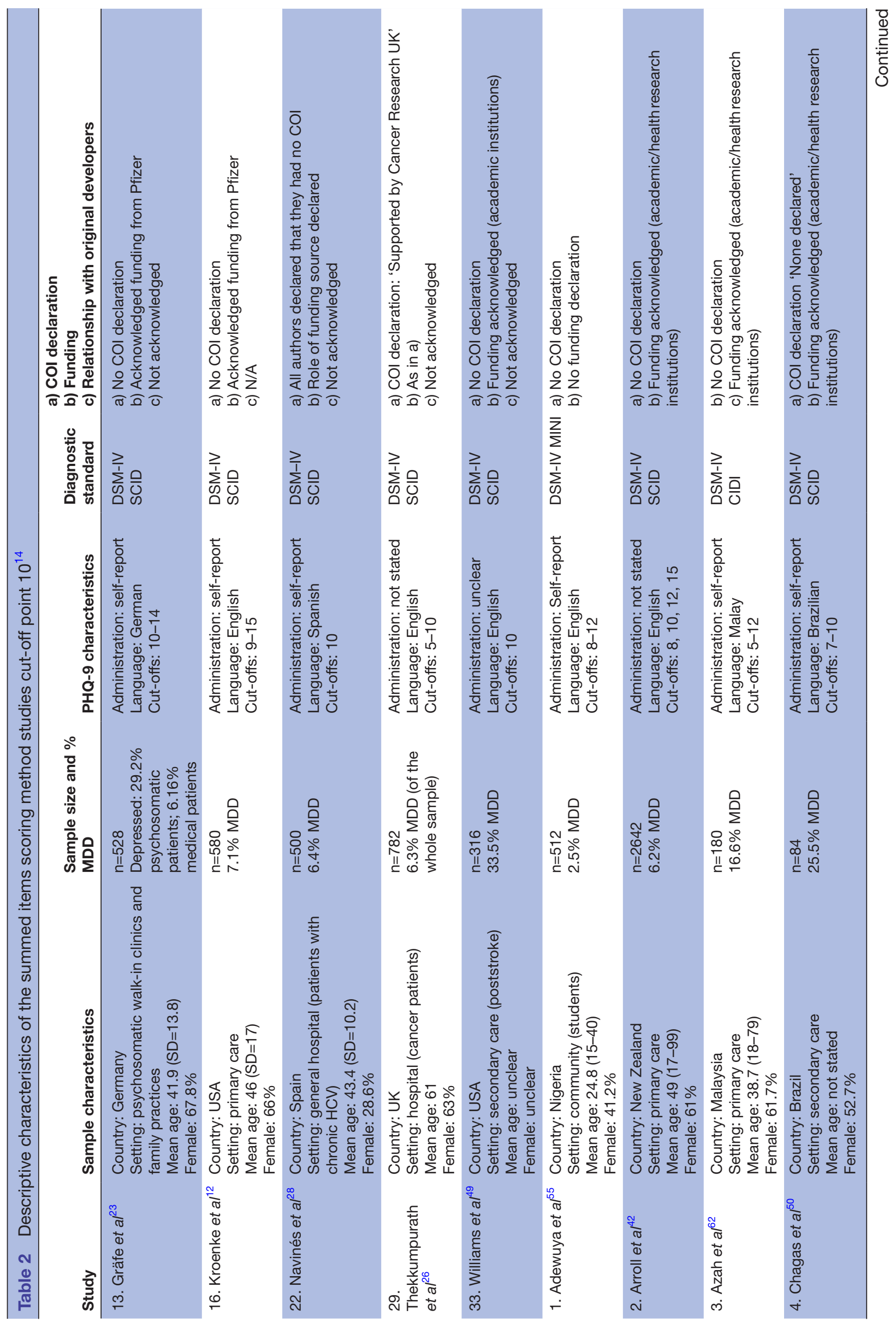




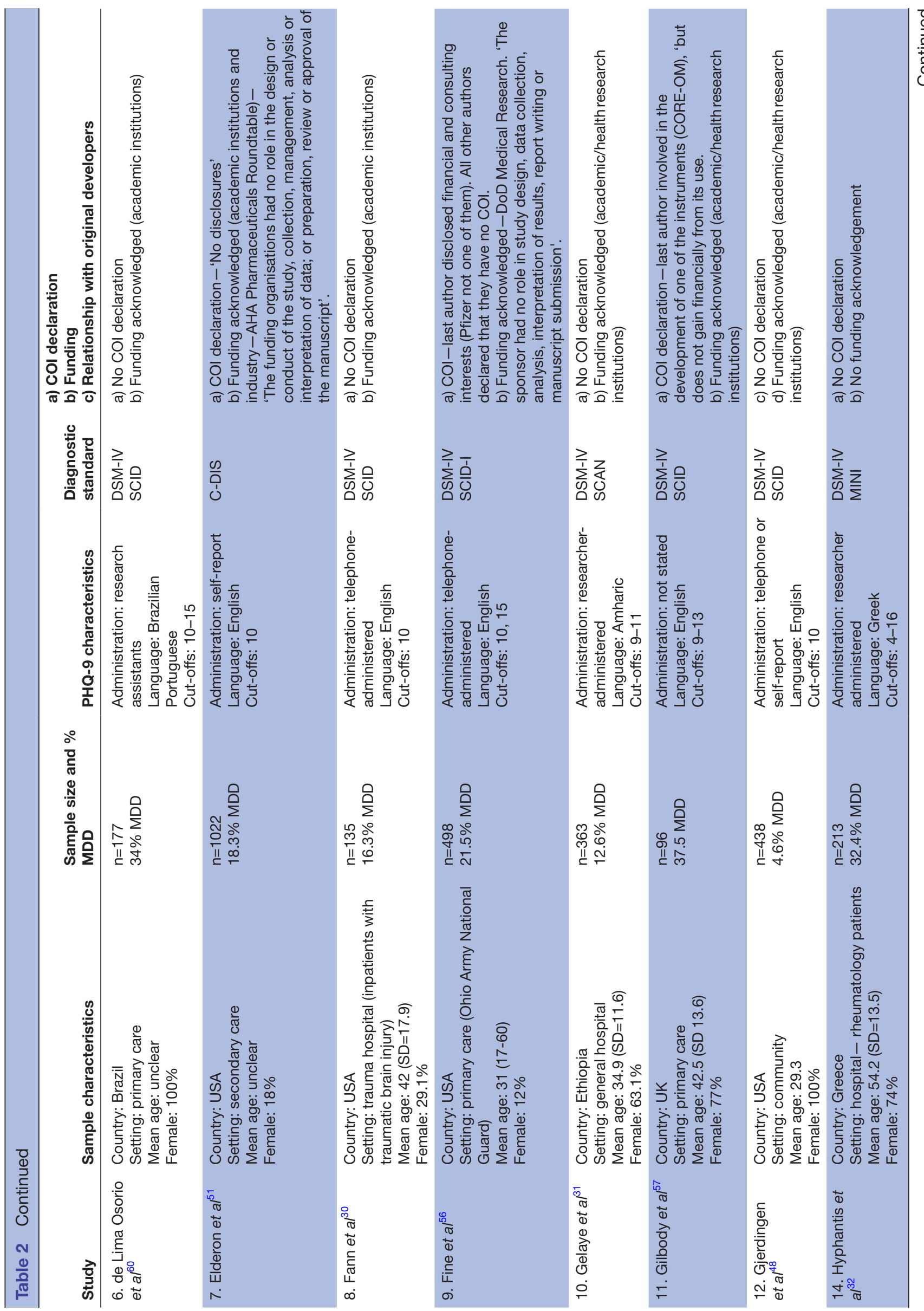




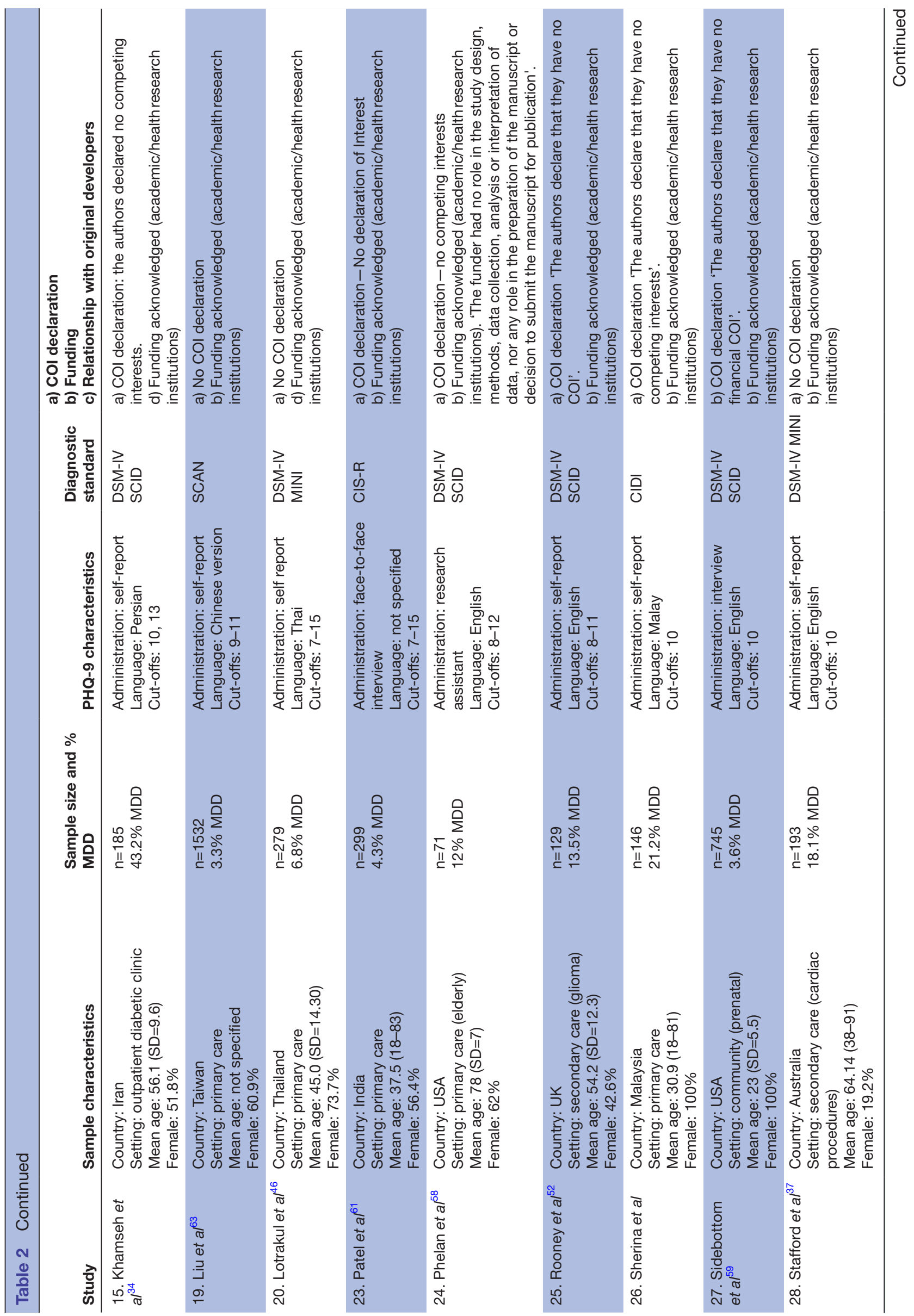




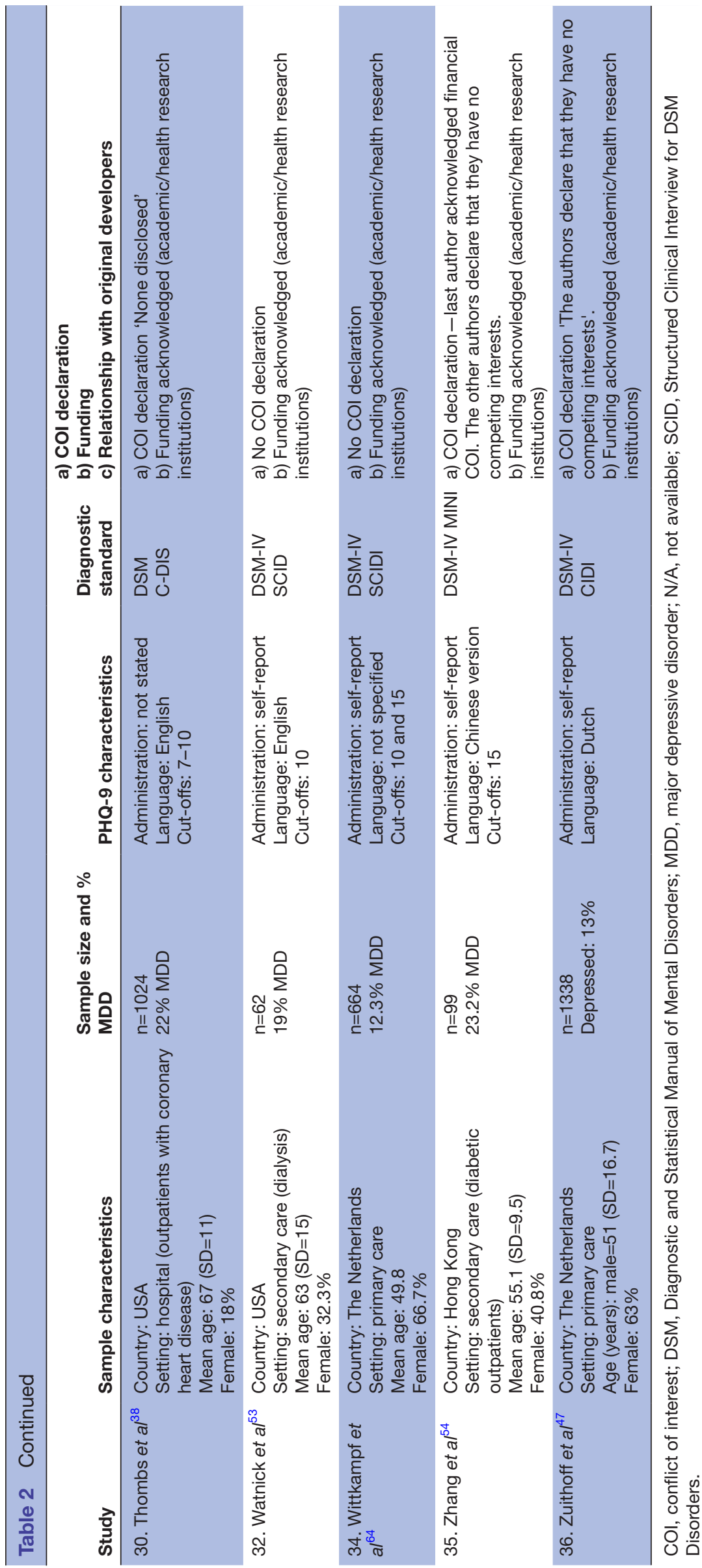

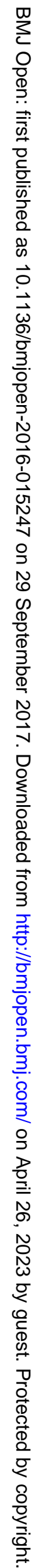


|

$\| \min \cdots \cdots$

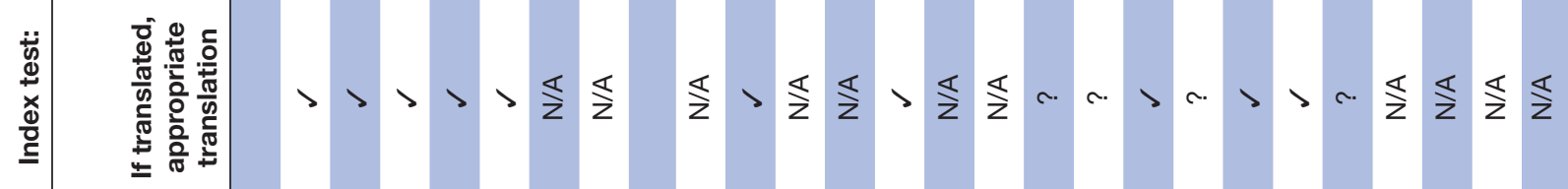

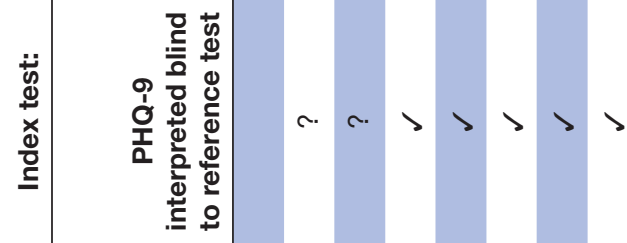

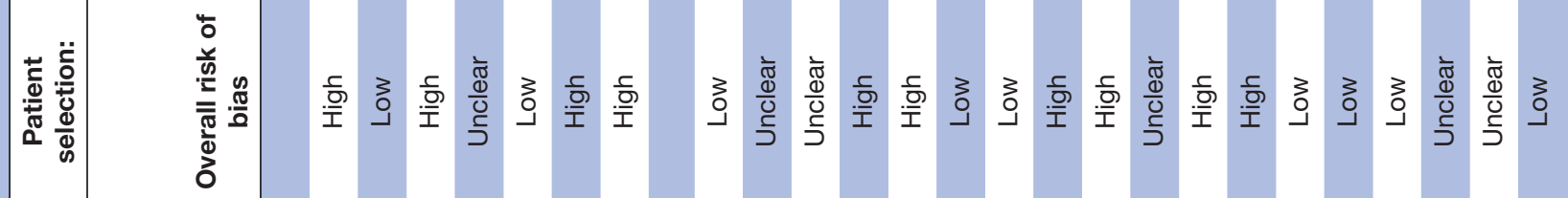




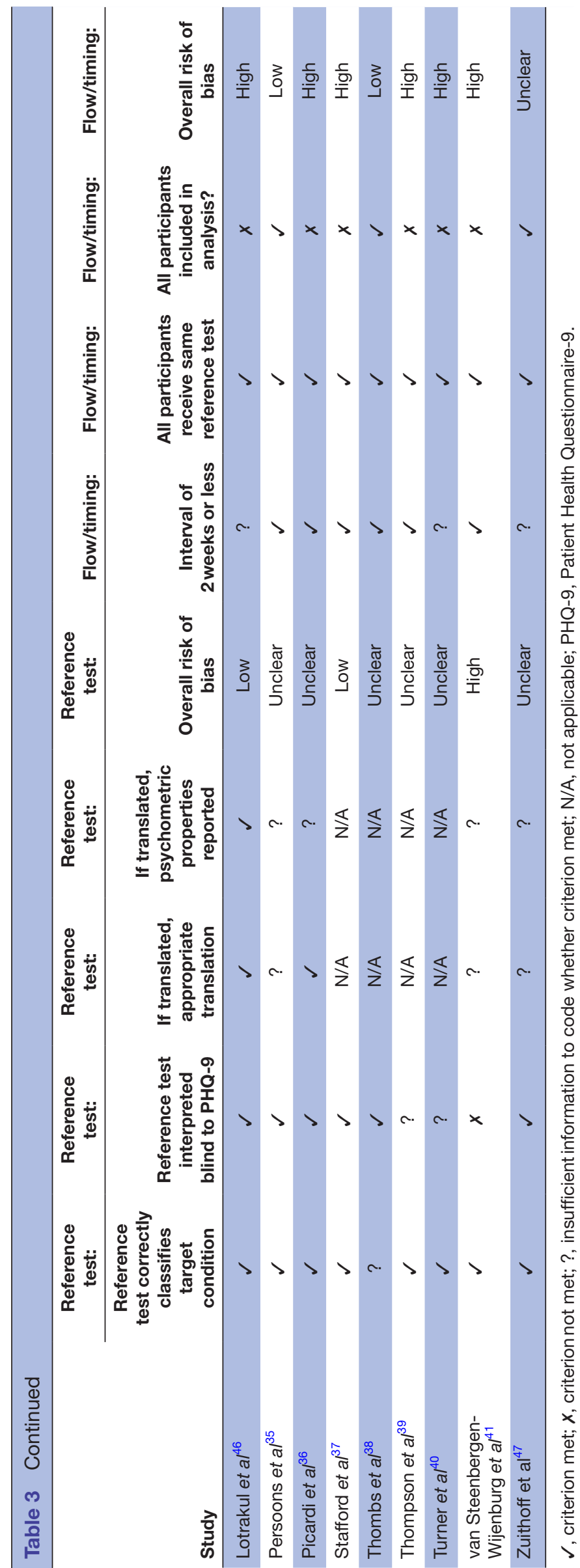




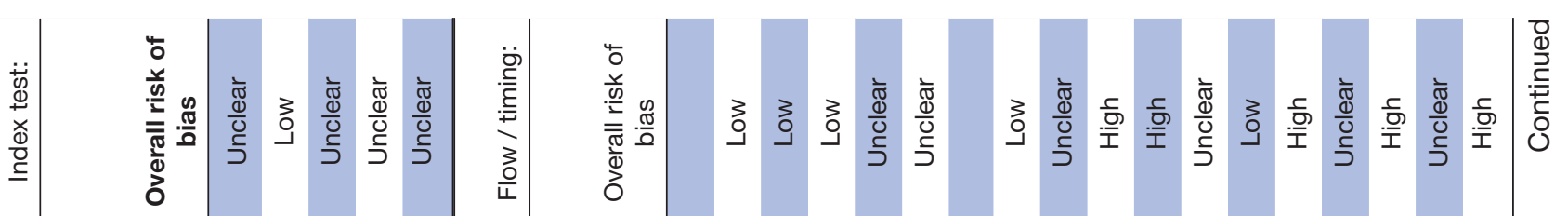

Miln:

ming

In

II

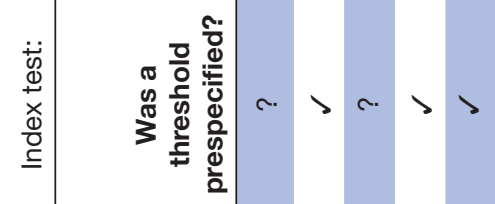

in

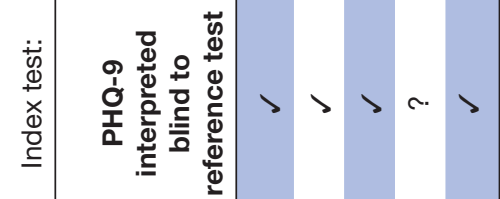

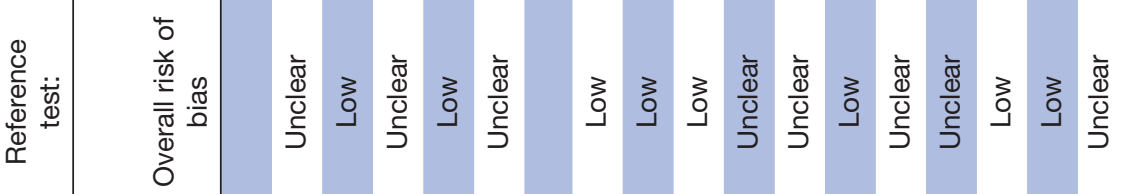

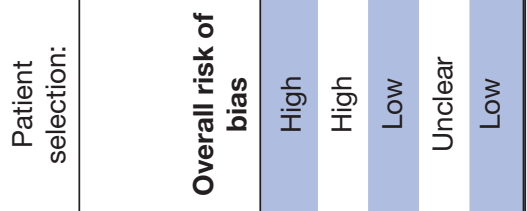

min

$>>>>>>>>>>>$

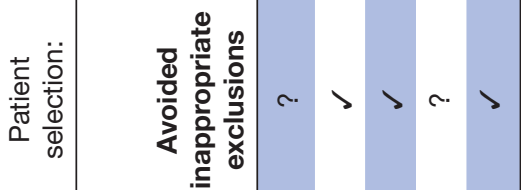

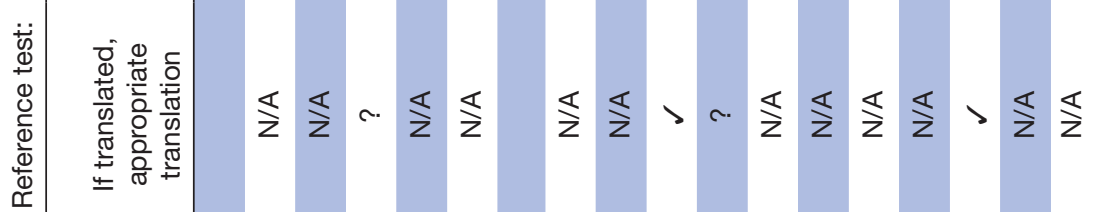

Min

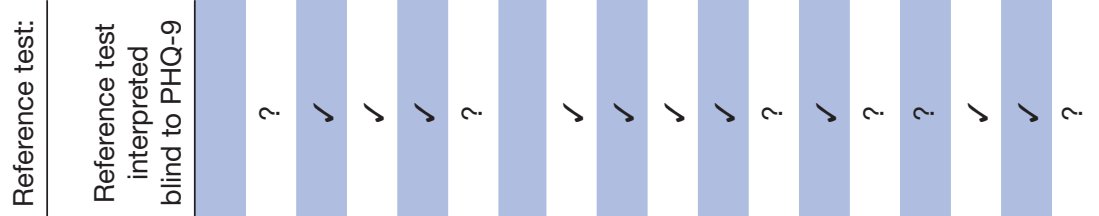

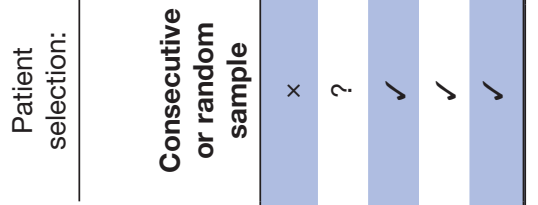

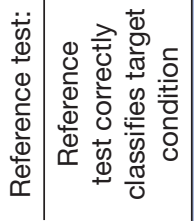

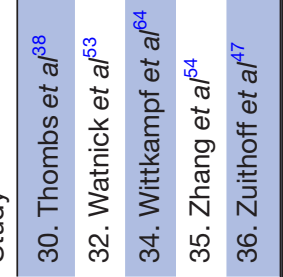

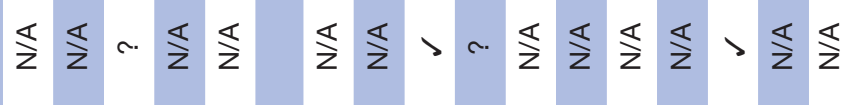

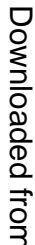

镸

$\frac{3}{3}$ 


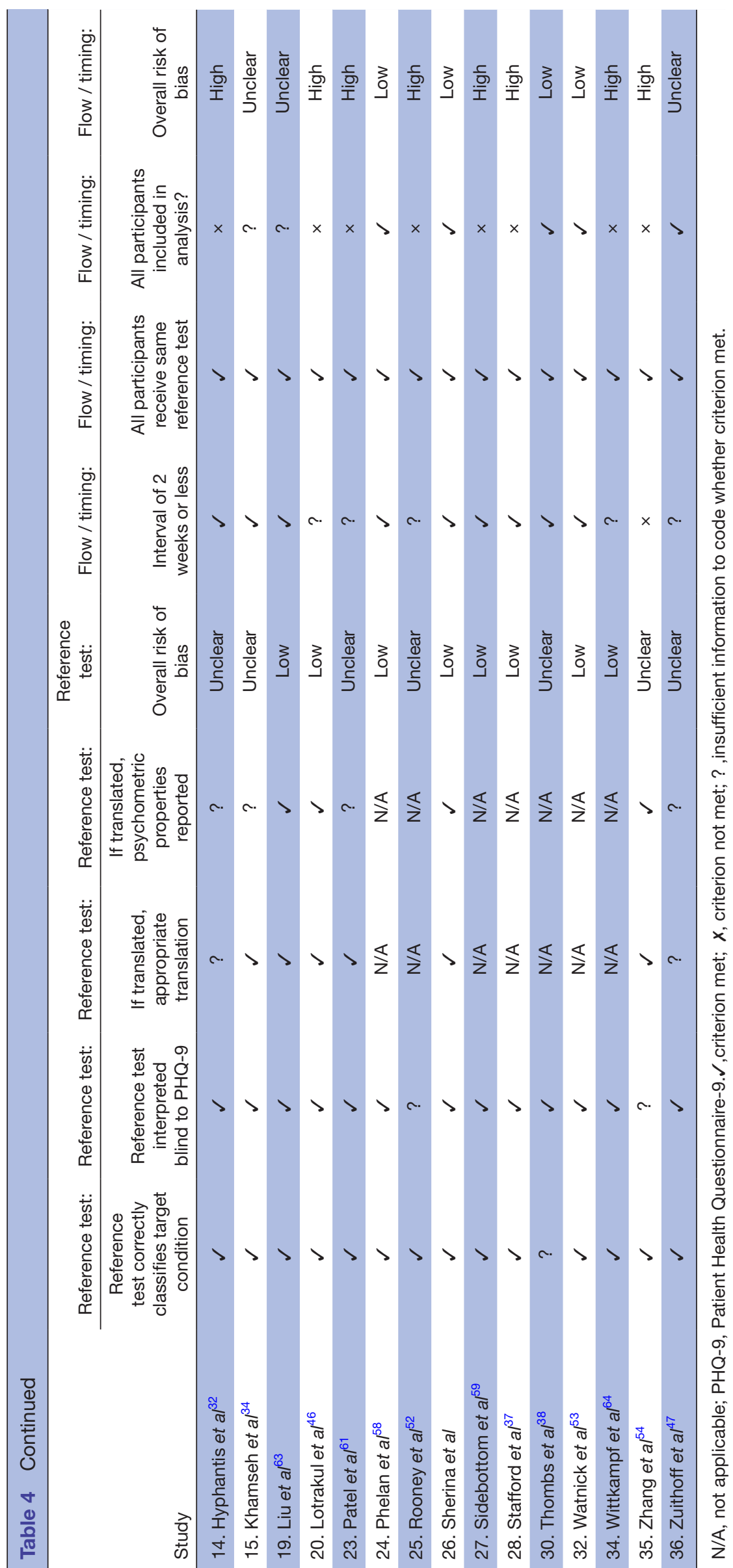



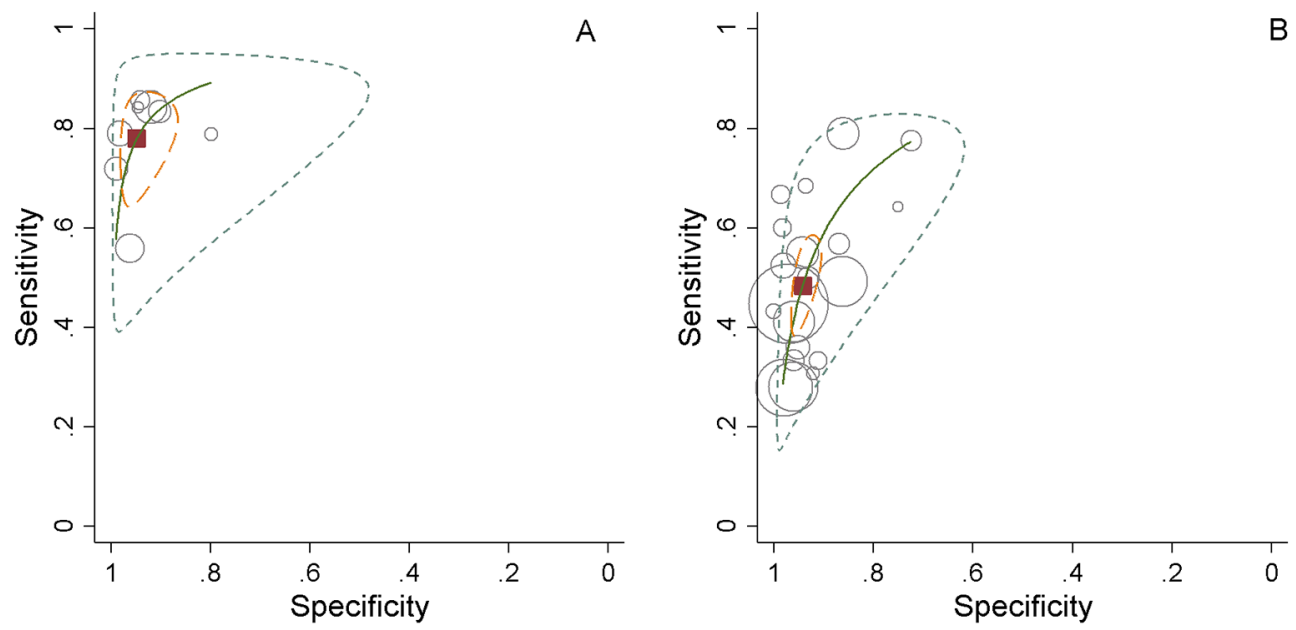

\begin{tabular}{|c|c|c|c|}
\hline 0 & Study estimate & Summary point & HSROC curve \\
\hline--- & $\begin{array}{l}95 \% \text { confidence } \\
\text { region }\end{array}$ & $\begin{array}{l}95 \% \text { prediction } \\
\text { region }\end{array}$ & \\
\hline
\end{tabular}

Figure 1 Patient Health Questionnaire-9 algorithm scoring method summary receiver operating characteristic plot for the diagnosis of major depressive disorder in allegiant studies (panel A) and non-allegiant studies (panel B). Pooled sensitivity and specificity estimates using a bivariate meta-analysis. HSROC, hierarchical receiver operating characteristic.

case-control design, avoid inappropriate exclusions). In the index test domain, the proportion of studies reporting that the PHQ-9 was conducted blind to the reference test was comparable between the two groups. There were differences in this domain for those studies using a translated version of the test. All non-English allegiant studies $(5 / 5)$ used an appropriately translated version of the PHQ-9, whereas just over a half of the non-allegiant studies reported this $(55 \%, 6 / 11)$. However, the majority of both sets of studies did not report details of psychometric properties of the translated version. For the reference test domain, nearly all studies in both groups were rated as using a reference test that would correctly classify the condition. While most allegiant studies reported that the reference test was interpreted blind to the PHQ-9 score $(86 \%, 6 / 7)$, this was reported in only $60 \%(12 / 20)$ of the non-allegiant studies.

The two sets of studies that used translated versions of the reference test were broadly comparable. There was a slight indication that the allegiant studies were more likely to use an appropriately translated version of the reference test and report data on the psychometric properties of the translated version, although the numbers for the translated comparison are very low. There were, however, some more notable differences on the flow and timing domain. Most allegiant studies ensured that the time between the index and reference test was under 2 weeks $(86 \%, 6 / 7)$ in comparison to $70 \%(14 / 20)$ of the non-allegiant studies. More allegiant studies met the criterion for 'all participants included in the analysis' $(57 \%, 4 / 7)$ than non-allegiant studies $(25 \%)$.

\section{Summed items scoring method (cut-off point 10 or above) Descriptive characteristics}

Table 2 presents the sample characteristics of the 31 PHQ-9 validation studies that reported the psychometric properties of the PHQ-9 at cut-off point 10 or above. Five of these studies were coauthored by the original developers of the instrument or acknowledged collaboration $^{12} 232649$ or were coauthored by the first author of a previous study that had also been coauthored by one of the developers. ${ }^{28}$ Twenty-six studies were conducted by independent researchers.

Three $(60 \%, 3 / 5)$ allegiant studies ${ }^{26} 2849$ and 11 non-allegiant studies $(42 \%, 11 / 26)^{30-32} 34373850-54$ were conducted in hospital settings.

Three $(60 \%, 3 / 5)$ allegiant studies ${ }^{122649}$ and 13 non-allegiant studies $(13 / 26)^{303738424851-5355-59}$ were conducted in English.

The mean prevalence of MDD in the allegiant group was $13.2 \%$ (range $6.1 \%-33.5 \%$ ) and in the non-allegiant group was $16.1 \%$ (range $2.5 \%-43.2 \%$ ). The mean age of patients in the allegiant group studies was 48.1 (range 41.9-61.0) and in the 26 non-allegiant studies that reported these data was 49.1 (range 23.0-78.0). The percentage of females in the allegiant studies that reported these data ${ }^{12} 232628$ was $56.3 \%$ (range 28.6\%$67.8 \%$ ) and in the non-allegiant group was $64.9 \%$ (range $12 \%-100 \%)$.

Three allegiant studies used the self-reported mode of administration and two of them did not specify how the PHQ-9 was administered. In nine non-allegiant studies $(34 \%, 9 / 26)$, the PHQ-9 was administered by the researcher. ${ }^{30-32485658-61}$ All allegiant studies used SCID as 
a gold standard; the non-allegiant studies used a wider range of gold standards including SCAN, CIDI, MINI, CIS-R, C-DIS, although the SCID was used in half of the studies $(50 \%, 13 / 26$ studies).

Three allegiant studies $(60 \%)$ did not include a conflict of interest statement. ${ }^{12} 2349$ Two of these studies ${ }^{12} 23$ acknowledged funding from Pfizer. None of the allegiant studies acknowledged collaboration or authorship of one of the developers of the PHQ-9.

Of the non-allegiant studies, $13(42 \%)$ did not include a conflict of interest statement. ${ }^{30-32} 3742464853556062-64$ Similar to the algorithm studies, the newer studies were more likely to include a conflict of interest statement. Funding was acknowledged by most studies (27/31) and most received funding from academic and/or health research institutions. One study ${ }^{57}$ acknowledged that the last author involved in the development of one of the instruments (CORE-OM), 'but does not gain financially from its use'. One study ${ }^{51}$ acknowledged funding from industry, AHA Pharmaceuticals Roundtable, but stated that 'the funding organisations had no role in the design or conduct of the study, collection, management, analysis or interpretation of data; or preparation, review or approval of the manuscript. Fine et al. disclosed that the last author had financial and consulting interests (Pfizer was not cited as one of them). ${ }^{56}$

\section{Diagnostic test accuracy}

Pooled sensitivity of allegiant studies was 0.87 (95\% CI 0.77 to 0.93 ), pooled specificity was 0.87 (95\% CI 0.76 to 0.94 ) and the pooled DOR was 49.31 (95\% CI 25.74 to 94.48 - - see table 5. Heterogeneity was moderate $\left(\mathrm{I}^{2}=55.1 \%\right)$. Figure 2 represents the sROCs for this group.

Pooled sensitivity of non-allegiant studies was 0.76 (95\% CI 0.67 to 0.83 ), pooled specificity was 0.88 (95\% CI 0.85 to 0.91 ) and the pooled DOR was 24.96 (95\% CI 14.81 to 42.08 ), approximately half that of the allegiant studies (table 2). Heterogeneity was high at $\mathrm{I}^{2}=81.5 \%$. Figure 2 represents the sROCs for this group.

The meta-regression for the studies using a cut-off point of 10 or above with allegiance status of the predictor showed that allegiance status was a significant predictor of the DOR $(\mathrm{p}=0.015)$ and explained $19.0 \%$ of observed heterogeneity.

\section{Quality assessment}

The results of the quality assessment using the QUADAS-2 are given in table 4 . For the patient selection domain, the two groups of studies were broadly comparable on two items (consecutive or random sample, avoid case-control design). However, all allegiant studies were rated as avoiding inappropriate exclusions $(5 / 5)$ in contrast to $58 \%(15 / 26)$ of the non-allegiant studies.

On the index test domain, there were a number of differences between the two groups of studies. More of the non-allegiant studies $(81 \%, 21 / 26)$ reported that the PHQ-9 was interpreted blind to the reference test compared with $60 \%(3 / 5)$ of the allegiant studies. All 

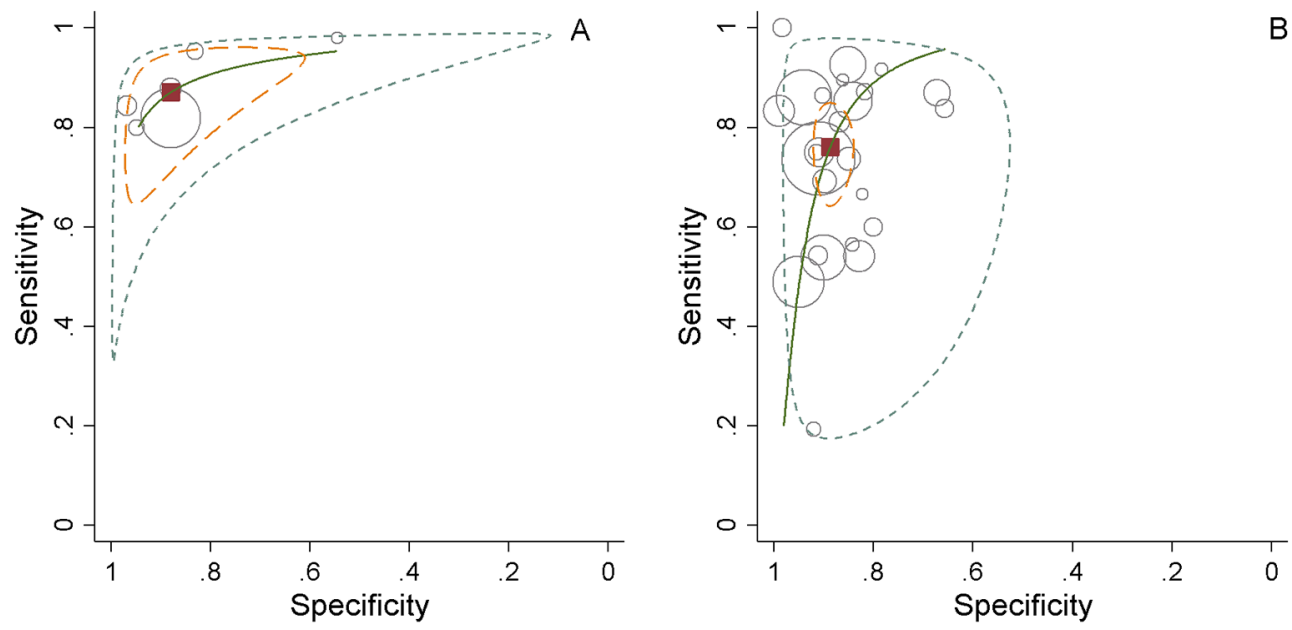

\begin{tabular}{|c|c|c|c|}
\hline$\circ$ & Study estimate & Summary point & HSROC curve \\
\hline--- & $\begin{array}{l}95 \% \text { confidence } \\
\text { region }\end{array}$ & $\begin{array}{l}95 \% \text { prediction } \\
\text { region }\end{array}$ & \\
\hline
\end{tabular}

Figure 2 Patient Health Questionnaire-9 summed items scoring method at cut-off point 10 summary receiver operating characteristic plot for diagnosis of major depressive disorder in allegiant studies (panel A) and non-allegiant studies (panel B). Pooled sensitivity and specificity using a bivariate meta-analysis. HSROC, hierarchical receiver operating characteristic.

$(5 / 5)$ allegiant studies were rated as prespecifying the threshold on the PHQ-9 compared with 73\% (19/26) of the non-allegiant studies. The two sets of studies were broadly comparable in terms of two items from the reference test domain (correctly classify target condition, reference test interpreted blind). Only one allegiant study used a translated version of the index test or reference test, so it is not possible to comment on differences between the two sets of studies in terms of these items from the index or reference test domains. For the flow and timing domain, the two groups of studies were broadly comparable for two of the criteria (interval of 2 weeks or less, all participants receive same reference test). However, fewer than half of the non-allegiant studies met the criterion for 'all participants included in the analysis' $(42 \%, 11 / 26)$, whereas all allegiant studies met this criterion.

\section{DISCUSSION}

This is to our knowledge the first systematic examination of a possible 'allegiance' or authorship effect in the validation of screening or case-finding psychological instrument for a common mental health disorder. We reviewed diagnostic validation studies of the PHQ-9, a widely used depression screening instrument. We found that allegiant studies reported higher sensitivity paired with similar specificity compared with non-allegiant studies. When entered as a covariate in meta-regression analyses, allegiance status was predictive of variation in the DOR for both the algorithm scoring method and the summeditem scoring method at a cut-off point of 10 or above.

Previous research has proposed several possible explanations for the allegiance effect. ${ }^{9-11}$ One possibility is the advertent bias that may serve to inflate the performance of a test when evaluated by those who have developed it. However, before concluding that the differences are due to this, it is important to explore and rule out alternative explanations. First, it is possible that any observed differences are a result of differences in study characteristics of the two sets of studies (eg, setting, clinical population). Second, differences in the methodological quality of the studies may also account for any differences. These possibilities are examined below.

\section{Difference in study characteristics as potential alternative explanations}

The two sets of studies were broadly comparable in terms of gender and the prevalence of depression, so these variables are unlikely to offer an explanation for the differences. While there were some indications from both sets of comparisons that the PHQ-9 may have been researcher-administered more often in the independent studies, it is not immediately clear how this would lead to lowered diagnostic performance.

The diagnostic meta-analyses of the PHQ- $9^{13} 14$ have shown that the sensitivity and DOR of the PHQ-9 tends to be lower in hospital settings for both algorithm and summed-item scoring methods. While the fact that proportionally more non-allegiant algorithm studies were conducted in secondary care could explain the lower sensitivity and DOR values in the algorithm studies, in the studies that reported the cut-off point of or above this would not be the case as proportionally more allegiant studies were conducted in hospital settings.

Similarly, differences in the proportions of studies using translated versions of the PHQ-9 are also unlikely to offer 
an obvious explanation of the difference in diagnostic performance, because in the algorithm set of studies more of the allegiant studies used a translated version of the test, but the proportions were in the opposite direction for the studies using a cut-off of 10 or above. We tested this by carrying out a sensitivity analysis restricting the sample to English studies and studies with adequate translation. The allegiance effect was still predictive of DOR variation between allegiance and non-allegiance studies variation in both algorithm $(\mathrm{p}=0.00)$ and summed item scoring at cut-off point of 10 meta-analyses $(\mathrm{p}=0.02)$.

A similar conclusion is also likely to apply to the age of the samples. There were more older adults studies in the non-allegiant than allegiant studies in the algorithm comparison. Depression could be more difficult to identify in older adults due to physical comorbidities that may present with similar symptomatology to depression and could account for the lower diagnostic performance in the non-allegiant studies. However, the non-allegiant samples in the studies that reported the psychometric properties at cut-off point 10 or above had younger samples than the allegiant studies, so this would not support this interpretation.

The SCID was used as the gold standard in nearly all allegiant studies. The fact that some non-allegiant studies used other gold standards could potentially explain the poorer psychometric properties of the PHQ-9 in these studies. The SCID is often regarded as the most valid of the available semi-structured interviews used in depression diagnostic validity studies as the reference standard. If we assume that this is the case and, furthermore, that the PHQ-9 is an accurate method of screening for depression, then the PHQ-9 may be more likely to agree with the SCID than other reference standards. However, when we carried out a sensitivity analysis restricting the sample to SCID-only studies, the allegiance effect was still predictive of DOR variation between allegiance and non-allegiance studies variation in both algorithm $(\mathrm{p}=0.01)$ and summed item scoring at cut-off point of 10 reviews $(\mathrm{p}=0.02)$.

\section{Differences in methodological quality as potential alternative explanations}

The quality of the studies was evaluated using the QUADAS-2. Although there were several potential methodological differences between the two groups of studies from the algorithm papers, not all of these offer obvious explanations of the observed differences and some are unlikely as explanations. For example, more allegiant studies ensured that the reference test was interpreted blind to the index test. This is unlikely to account for the observed differences, because a lack of blinding is typically associated with artificially increased diagnostic performance, which is in the opposite direction to the pattern of results observed here. The impact of some other differences is less clear-cut. For example, a higher number of the non-allegiant studies met the criterion for consecutive referrals. For this to provide an explanation of the observed differences, the non-consecutive nature of the referrals in the studies by those who had developed the PHQ-9 would need to have led to the overinclusion of true positives or underinclusion of false negatives given that these studies tended to report higher sensitivity relative to the non-allegiant studies (and vice versa for the independent studies). It is not immediately obvious how this would occur. The allegiant studies were more likely to have met the criterion of 'included all participants in the analysis'. It is possible that the greater loss of participants from the non-allegiant studies may have artificially reduced the observed diagnostic accuracy, although, again, it is not immediately obvious how this would have affected the true positive and false negative rates. Although there is not an obvious explanation of how these differences in methodological quality could account for the observed differences in diagnostic performance, it is important to recognise that they cannot on that basis be ruled out.

There are, however, two differences in methodological quality among the algorithm studies that are clearer potential alternative explanations. The higher rate of appropriate translations among the allegiant studies is potentially important, because lower diagnostic estimates may be expected from studies that have poorly translated versions of the index test. In the flow and timing domain, more allegiant studies ensured that there was a less than 2-week interval between the index and reference test. This is consistent with lower diagnostic performance in the non-allegiant studies: as the interval increases it is likely that depression status may change and this would lead to lower levels of agreement between the index test and the reference test.

There were also differences on some quality assessment items between the two sets of studies in the summed item scoring method comparison. The threshold was reported as prespecified in all allegiant studies in contrast to approximately three-quarters of the non-allegiant studies. On the face of it, this is unlikely to explain the observed differences, because the use of a prespecified cut-off point is likely to be associated with lower not higher diagnostic test performance. One possibility, however, is that studies that performed poorly at this cut-off point were less likely to be reported by those who had developed the measure. As discussed in more detail in the 'Limitations' section, we were unable to explore this possibility through the use of formal tests for publication bias.

All allegiant studies avoided inappropriate exclusions compared with approximately half of the non-allegiant studies. While this is a potential alternative explanation of the differences, it is not immediately obvious how this would explain the differences in diagnostic performance between the two sets of studies. Fewer than half of the non-allegiant studies met the criterion for 'all participants included in the analysis', in contrast to all of the allegiant studies met this criterion, but again this difference should usually work against the inclusive studies, not those excluding cases. More of the non-allegiant studies reported that the PHQ-9 was interpreted blind to the 
reference test. This does offer a potential explanation, because the absence of blinding may artificially inflate diagnostic accuracy.

\section{LIMITATIONS}

The results of this review need to be viewed in light of the limitations of the primary studies that contributed to the review and the review itself. An important consideration is to establish whether any observed differences between the diagnostic performance of the non-allegiant and allegiant studies are better accounted for by study characteristic or methodological differences. Caution, however, is needed in interpreting any differences, because of the small number of allegiant studies in both the algorithm and cut-off 10 or above comparisons. The small number of allegiant studies also meant that we were also unable to explore the potential role of publication bias in the non-allegiant and allegiant studies. At least 10 studies are required to use standard methods of examining publication bias, but the number of allegiant studies in both the algorithm and cut-off 10 or above comparisons were fewer than this. Papers published from August 2013 onwards are not covered in the literature search used and so it potentially misses some more recent studies that would be eligible for inclusion, although it is unlikely that many, if any, new allegiant studies have been published since.

\section{CONCLUSIONS AND IMPLICATIONS FOR FURTHER RESEARCH}

The aims of the review was to investigate whether an allegiance effect is found that leads to an increased diagnostic performance in diagnostic validation studies that were conducted by teams connected to the original developers of the PHQ-9. Our analyses showed that diagnostic studies conducted by independent/ non-allegiant researchers had lower sensitivity paired with similar specificity compared with studies that were classified as allegiant. This conclusion held for both the algorithm and cut-off 10 or above studies. We explored a range of possible alternative explanations for the observed allegiance effect including both differences in study characteristics and study quality. A number of potential differences were found, although for some of these it is not clear how they would necessarily account for the observed differences. However, there were a number of differences that offered potential alternative explanations unconnected to allegiance effects. In the algorithm studies, the studies rated as allegiant were also more likely to use an appropriate translation of the PHQ-9 and were also more likely to ensure that the index and reference test were conducted within 2 weeks of each other, both of which may be associated with an improvement in observed diagnostic performance of an instrument. The majority of studies in both meta-analyses did not provide clear statements about potential conflict of interest and/or funding; however, the newer studies were more likely to provide such statements, which may reflect increasing transparency in this area of research.

We cannot, therefore, conclude that allegiance effects are present in studies examining the diagnostic performance of the PHQ-9; but nor can we rule them out. Conflicts of interest are an important area of investigation in medical and behavioural research, particularly due to concerns about trial results being influenced by industry sponsorship. Future diagnostic validity in this area should as a matter of routine present clear statements about potential conflicts of interest and funding, particularly relating to the development of the instrument under evaluation. Future meta-analyses of diagnostic validation studies of psychological measures should routinely evaluate the impact of researcher allegiance in the primary studies examined in the meta-analysis.

Acknowledgements One of the authors of this paper (SG) was supported by the NIHR Collaboration for Leadership in Applied Health Research and Care Yorkshire and Humber (NIHR CLAHRC YH). The views and opinions expressed are those of the author(s), and not necessarily those of the NHS, the NIHR or the Department of Health.

Contributors LM led on all stages of the review and is the guarantor. We used an established database of diagnostic validation studies of the PHQ-9 (Manea et al., 2015; Moriarty et al., 2015). SG provided expert advice on methodology and approaches to assessment of the evidence base. AM carried out the literature searches, screened the studies, extracted data and assessed the quality of the included studies for one of the systematic reviews (Moriarty et al., 2015). LM carried out the literature searches, screened the studies, extracted data and assessed the quality of the included studies for the other systematic review (Manea et al., 2015), analysed the data for both systematic reviews and drafted the report. JB involved in the development of the study, wrote the introduction section of the review and contributed to the production of the final report. DM supervised the quality assessment, methodology and approaches to evidence synthesis, provided senior advice and supported throughout and contributed to the production of the final report. All parties were involved in drafting and/or commenting on the report.

Funding LM was an NIHR Clinical Lecturer when this research was carried out. The NIHR had no role in the study design, methods, data collection, analysis or interpretation of data, nor any role in the preparation of the manuscript or decision to submit the manuscript for publication.

Competing interests None declared.

Provenance and peer review Not commissioned; externally peer reviewed.

Data sharing statement № additional data are available.

Open Access This is an Open Access article distributed in accordance with the terms of the Creative Commons Attribution (CC BY 4.0) license, which permits others to distribute, remix, adapt and build upon this work, for commercial use, provided the original work is properly cited. See: http://creativecommons.org/ licenses/by/4.0/

(C) Article author(s) (or their employer(s) unless otherwise stated in the text of the article) 2017. All rights reserved. No commercial use is permitted unless otherwise expressly granted.

\section{REFERENCES}

1. Luborsky L, Diguer L, Seligman DA, et al. The researcher's own therapy allegiances: a "Wild Card" in comparisons of treatment efficacy. Clin Psychol: Sci Pract 2006;6:95-106.

2. Dragioti E, Dimoliatis I, Evangelou E. Disclosure of researcher allegiance in meta-analyses and randomised controlled trials of psychotherapy: a systematic appraisal. BMJ Open 2015;5:e007206. 
3. Munder T, Brütsch $\mathrm{O}$, Leonhart R, et al. Researcher allegiance in psychotherapy outcome research: an overview of reviews. Clin Psychol Rev 2013;33:501-11.

4. Winter DA. "Editorial." Routledge, 2010.

5. McLeod J. "Taking allegiance seriously-implications for research policy and practice,". Eur J Psychother Couns 2010;12.

6. Staines GL, Cleland CM. Bias in meta-analytic estimates of the absolute efficacy of psychotherapy. Rev. Gen. Psychol 2007;11:329-47

7. Markman KD, Hirt ER. Social Prediction and the "Allegiance Bias". Soc Cogn 2002;20:58-86.

8. Walters GD. The psychological inventory of criminal thinking styles and psychopathy checklist: screening version as incrementally valid predictors of recidivism. Law Hum Behav 2009;33:497-505.

9. Singh JP, Grann M, Fazel S. Authorship bias in violence risk assessment? A systematic review and meta-analysis. PLoS One 2013;8:e72484.

10. Blair PR, Marcus DK, Boccaccini MT. Is there an allegiance effect for assessment instruments? actuarial risk assessment as an exemplar. Clin PsycholSci Pract 2008;15:346-60.

11. Lilienfeld SO, Jones MK. Allegiance effects in assessment: unresolved questions, potential explanations, and constructive remedies. Clin PsycholSci Pract 2008;15:361-5.

12. Kroenke K, Spitzer RL, Williams JB. The PHQ-9: validity of a brief depression severity measure. J Gen Intern Med 2001;16:606-13.

13. Manea L, Gilbody S, McMillan D. A diagnostic meta-analysis of the patient health questionnaire-9 (PHQ-9) algorithm scoring method as a screen for depression. Gen Hosp Psychiatry 2015;37:67-75.

14. Moriarty AS, Gilbody S, McMillan D, et al. Screening and case finding for major depressive disorder using the Patient Health Questionnaire (PHQ-9): a meta-analysis. Gen Hosp Psychiatry 2015;37:567-76.

15. Whiting PF, Rutjes AW, Westwood ME, et al. QUADAS-2: a revised tool for the quality assessment of diagnostic accuracy studies. Ann Intern Med 2011;155:529-36.

16. Mann R, Hewitt CE, Gilbody SM. Assessing the quality of diagnostic studies using psychometric instruments: applying QUADAS. Soc Psychiatry Psychiatr Epidemiol 2009;44:300-7.

17. Reitsma JB, Glas AS, Rutjes AW, et al. Bivariate analysis of sensitivity and specificity produces informative summary measures in diagnostic reviews. J Clin Epidemiol 2005;58:982-90.

18. University of York. NHS Centre for Reviews and Dissemination. Systematic reviews : CRD's guidance for undertaking reviews in health care: CRD, University of York, 2009.

19. Walter SD. Properties of the summary receiver operating characteristic (SROC) curve for diagnostic test data. Stat Med 2002;21:1237-56.

20. Lijmer JG, Bossuyt PM, Heisterkamp SH. Exploring sources of heterogeneity in systematic reviews of diagnostic tests. Stat Med 2002;21:1525-37.

21. Thompson SG, Higgins JP. How should meta-regression analyses be undertaken and interpreted? Stat Med 2002;21:1559-73.

22. Diez-Quevedo C, Rangil T, Sanchez-Planell L, et al. Validation and utility of the patient health questionnaire in diagnosing mental disorders in 1003 general hospital Spanish inpatients. Psychosom Med 2001;63:679-86.

23. Gräfe K, Zipfel S, Herzog W, et al. Screening psychischer Störungen mit dem "Gesundheitsfragebogen für Patienten (PHQ-D)". Diagnostica 2004;50:171-81.

24. Löwe B, Spitzer RL, Gräfe K, et al. Comparative validity of three screening questionnaires for DSM-IV depressive disorders and physicians' diagnoses. J Affect Disord 2004;78:131-40.

25. Spitzer RL, Kroenke K, Williams JB. Validation and utility of a selfreport version of PRIME-MD: the PHQ primary care study. Primary Care Evaluation of Mental Disorders. Patient Health Questionnaire. JAMA 1999;282:1737-44.

26. Thekkumpurath P, Walker J, Butcher I, et al. Screening for major depression in cancer outpatients: the diagnostic accuracy of the 9-item patient health questionnaire. Cancer 2011;117:218-27.

27. Muramatsu K, Miyaoka H, Kamijima K, et al. The patient health questionnaire, Japanese version: validity according to the miniinternational neuropsychiatric interview-plus. Psychol Rep 2007;101(3 Pt 1):952-60.

28. Navinés R, Castellví P, Moreno-España J, et al. Depressive and anxiety disorders in chronic hepatitis $\mathrm{C}$ patients: reliability and validity of the Patient Health Questionnaire. J Affect Disord 2012;138:343-51.

29. Eack SM, Greeno CG, Lee BJ. Limitations of the patient health questionnaire in identifying anxiety and depression: many cases are undetected. Res Soc Work Pract 2006;16:625-31.
30. Fann JR, Bombardier CH, Dikmen S, et al. Validity of the patient health questionnaire- 9 in assessing depression following traumatic brain injury. J Head Trauma Rehabil 2005;20:501-11.

31. Gelaye B, Williams MA, Lemma S, et al. Validity of the patient health questionnaire- 9 for depression screening and diagnosis in East Africa. Psychiatry Res 2013;210:653-61.

32. Hyphantis T, Kotsis K, Voulgari PV, et al. Diagnostic accuracy, internal consistency, and convergent validity of the Greek version of the patient health questionnaire 9 in diagnosing depression in rheumatologic disorders. Arthritis Care Res 2011;63:1313-21.

33. Inagaki $\mathrm{M}$, Ohtsuki $\mathrm{T}$, Yonemoto $\mathrm{N}$, et al. Validity of the patient health questionnaire (PHQ)-9 and PHQ-2 in general internal medicine primary care at a Japanese rural hospital: a cross-sectional study. Gen Hosp Psychiatry 2013;35:592-7.

34. Khamseh ME, Baradaran HR, Javanbakht A, et al. Comparison of the CES-D and PHQ-9 depression scales in people with type 2 diabetes in Tehran, Iran. BMC Psychiatry 2011;11:61.

35. Persoons P, Luyckx K, Desloovere C, et al. Anxiety and mood disorders in otorhinolaryngology outpatients presenting with dizziness: validation of the self-administered PRIME-MD patient health questionnaire and epidemiology. Gen Hosp Psychiatry 2003;25:316-23.

36. Picardi A, Adler DA, Abeni D, et al. Screening for depressive disorders in patients with skin diseases: a comparison of three screeners. Acta Derm Venereol 2005;85:414-9.

37. Stafford L, Berk M, Jackson HJ. Validity of the hospital anxiety and depression scale and patient health questionnaire- 9 to screen for depression in patients with coronary artery disease. Gen Hosp Psychiatry 2007;29:417-24.

38. Thombs BD, Ziegelstein RC, Whooley MA. Optimizing detection of major depression among patients with coronary artery disease using the patient health questionnaire: data from the heart and soul study. $J$ Gen Intern Med 2008;23:2014-7.

39. Thompson AW, Liu H, Hays RD, et al. Diagnostic accuracy and agreement across three depression assessment measures for Parkinson's disease. Parkinsonism Relat Disord 2011;17:40-5.

40. Turner A, Hambridge J, White J, et al. Depression screening in stroke: a comparison of alternative measures with the structured diagnostic interview for the diagnostic and statistical manual of mental disorders, fourth edition (major depressive episode) as criterion standard. Stroke 2012;43:1000-5.

41. van Steenbergen-Weijenburg KM, de Vroege L, Ploeger RR, et al. Validation of the PHQ-9 as a screening instrument for depression in diabetes patients in specialized outpatient clinics. BMC Health Serv Res 2010;10:235.

42. Arroll B, Goodyear-Smith F, Crengle S, et al. Validation of PHQ-2 and $\mathrm{PHQ}-9$ to screen for major depression in the primary care population. Ann Fam Med 2010;8:348-53.

43. Ayalon L, Goldfracht M, Bech P. 'Do you think you suffer from depression?' Reevaluating the use of a single item question for the screening of depression in older primary care patients. Int J Geriatr Psychiatry 2010;25:497-502.

44. Henkel V, Mergl R, Kohnen R, et al. Use of brief depression screening tools in primary care: consideration of heterogeneity in performance in different patient groups. Gen Hosp Psychiatry 2004;26:190-8.

45. Lamers F, Jonkers CC, Bosma H, et al. Summed score of the patient health questionnaire- 9 was a reliable and valid method for depression screening in chronically ill elderly patients. J Clin Epidemiol 2008;61:679-87.

46. Lotrakul M, Sumrithe S, Saipanish R. Reliability and validity of the Thai version of the PHQ-9. BMC Psychiatry 2008;8:46

47. Zuithoff NP, Vergouwe $Y$, King M, et al. The patient health questionnaire- 9 for detection of major depressive disorder in primary care: consequences of current thresholds in a crosssectional study. BMC Fam Pract 2010;11:98.

48. Gjerdingen D, Crow S, McGovern P, et al. Postpartum depression screening at well-child visits: validity of a 2-question screen and the PHQ-9. Ann Fam Med 2009;7:63-70.

49. Williams LS, Brizendine EJ, Plue L, et al. Performance of the PHQ-9 as a screening tool for depression after stroke. Stroke 2005;36:635-8.

50. Chagas $\mathrm{MH}$, Tumas V, Rodrigues GR, et al. Validation and internal consistency of Patient Health Questionnaire-9 for major depression in Parkinson's disease. Age Ageing 2013;42:645-9.

51. Elderon L, Smolderen KG, Na B, et al. Accuracy and prognostic value of American Heart Association: recommended depression screening in patients with coronary heart disease: data from the Heart and Soul Study. Circ Cardiovasc Qual Outcomes 2011;4:533-40.

52. Rooney AG, McNamara S, Mackinnon M, et al. Screening for major depressive disorder in adults with cerebral glioma: an initial validation of 3 self-report instruments. Neuro Oncol 2013;15:122-9. 
53. Watnick S, Wang PL, Demadura T, et al. Validation of 2 depression screening tools in dialysis patients. Am J Kidney Dis 2005;46:919-24.

54. Zhang $\mathrm{Y}$, Ting R, Lam M, et al. Measuring depressive symptoms using the Patient Health Questionnaire- 9 in Hong Kong Chinese subjects with type 2 diabetes. J Affect Disord 2013;151:660-6.

55. Adewuya AO, Ola BA, Afolabi OO. Validity of the patient health questionnaire (PHQ-9) as a screening tool for depression amongst Nigerian university students. J Affect Disord 2006;96:89-93.

56. Fine TH, Contractor AA, Tamburrino M, et al. Validation of the telephone-administered PHQ-9 against the in-person administered SCID-I major depression module. J Affect Disord 2013;150:1001-7.

57. Gilbody S, Richards D, Brealey S, et al. Screening for depression in medical settings with the Patient Health Questionnaire (PHQ): a diagnostic meta-analysis. J Gen Intern Med 2007;22:1596-602.

58. Phelan E, Williams B, Meeker K, et al. A study of the diagnostic accuracy of the PHQ-9 in primary care elderly. BMC Fam Pract 2010;11:63.

59. Sidebottom AC, Harrison PA, Godecker A, et al. Validation of the patient health questionnaire (PHQ)-9 for prenatal depression screening. Arch Womens Ment Health 2012;15:367-74.
60. de Lima Osório F, Vilela Mendes A, Crippa JA, et al. Study of the discriminative validity of the PHQ-9 and PHQ-2 in a sample of Brazilian women in the context of primary health care. Perspect Psychiatr Care 2009;45:216-27.

61. Patel V, Araya R, Chowdhary N, et al. Detecting common mental disorders in primary care in India: a comparison of five screening questionnaires. Psychol Med 2008;38.

62. Azah MNN, Shah MEM, Juwita S, et al. Validation of the Malay version brief patient health questionnaire (PHQ-9) among adult attending family medicine clinics. Int Med J 2005

63. Liu SI, Yeh ZT, Huang HC, et al. Validation of patient health questionnaire for depression screening among primary care patients in Taiwan. Compr Psychiatry 2011;52:96-101.

64. Wittkampf K, van Ravesteijn H, Baas K, et al. The accuracy of patient health questionnaire-9 in detecting depression and measuring depression severity in high-risk groups in primary care. Gen Hosp Psychiatry 2009;31:451-9. 